\title{
Erratum: Stress tensor and current correlators of interacting conformal field theories in $2+1$ dimensions: fermionic Dirac matter coupled to $U(1)$ gauge field
}

Yejin Huh ${ }^{a, b}$ and Philipp Strack ${ }^{a, c, 1}$

${ }^{a}$ Department of Physics, Harvard University, Cambridge MA 02138, U.S.A.

${ }^{b}$ Department of Physics, University of Toronto, Ontario M5S 1A7, Canada

${ }^{c}$ Institut für Theoretische Physik, Universität zu Köln, D-50937 Cologne, Germany

E-mail: yhuh@physics.utoronto.ca, pstrack@physics.harvard.edu

ERratum to: JHEP01(2015)147

ARXIV EPRINT: 1410.1902

Here we fix a sign error which affects results of two diagrams and therefore the $C_{T}$ value. The corrected Feynman rule is represented in the figure below.

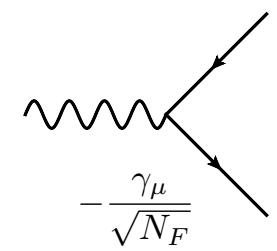

Figure 1. Corrected Feyman rule from figure 1 of the main text.

This changes the signs of diagrams 4 and 6 of the $C_{T}$ evaluation.

Therefore the final expression for $C_{T}$ is modified to

$$
\begin{aligned}
\frac{C_{T}}{N_{F}} & =\frac{1}{256}\left(1+\frac{1}{N_{F}}\left({\tilde{C_{T}}}^{(1)}+\frac{104}{15 \pi^{2}}\right)\right) \\
& =\frac{1}{256}\left(1+\frac{0.28701185900024704065}{N_{F}}\right) .
\end{aligned}
$$

${ }^{1}$ http://users.physics.harvard.edu/ p pstrack. 


$$
\begin{aligned}
T_{\mu \nu \lambda \rho}^{(4)}(p)= & N_{F} \operatorname{Tr}\left[\int_{\boldsymbol{k}, \boldsymbol{q}} \frac{1}{4} \gamma_{\lambda}(2 \boldsymbol{k}+\boldsymbol{p})_{\rho} \frac{(\boldsymbol{k}+\boldsymbol{p})_{a} \gamma_{a}}{(\boldsymbol{k}+\boldsymbol{p})^{2}} \frac{\gamma_{\epsilon}}{\sqrt{N_{F}}} \frac{(\boldsymbol{k}+\boldsymbol{p}+\boldsymbol{q})_{b} \gamma_{b}}{(\boldsymbol{k}+\boldsymbol{p}+\boldsymbol{q})^{2}} \frac{\gamma_{\mu}}{2 \sqrt{N_{F}}} \frac{k_{c} \gamma_{c}}{k^{2}} \frac{16}{q}\left(\delta_{\nu \epsilon}-\frac{q_{\nu} q_{\epsilon}}{q^{2}}\right)\right]+(\text { perm } 2) \\
T_{\mu \nu \lambda \rho}^{(6)}(p)= & -\int_{\boldsymbol{q}} N_{F} \operatorname{Tr}\left[\int_{\boldsymbol{k}} \frac{\gamma_{\rho}}{2 \sqrt{N_{F}}} \frac{k_{a} \gamma_{a}}{k^{2}} \frac{\gamma_{\kappa}}{\sqrt{N_{F}}} \frac{(\boldsymbol{k}+\boldsymbol{q})_{b} \gamma_{b}}{(\boldsymbol{k}+\boldsymbol{q})^{2}}\right] \frac{16}{q}\left(\delta_{\kappa \epsilon}-\frac{q_{\kappa} q_{\epsilon}}{q^{2}}\right) \frac{16}{|\boldsymbol{p}+\boldsymbol{q}|}\left(\delta_{\lambda \alpha}-\frac{(\boldsymbol{p}+\boldsymbol{q})_{\lambda}(\boldsymbol{p}+\boldsymbol{q})_{\alpha}}{(\boldsymbol{p}+\boldsymbol{q})^{2}}\right) \\
& \left(N_{F} \operatorname{Tr}\left[\int_{\boldsymbol{l}} \frac{1}{4} \gamma_{\mu}(\boldsymbol{p}+2 \boldsymbol{l})_{\nu} \frac{l_{c} \gamma_{c}}{l^{2}} \frac{\gamma_{\alpha}}{\sqrt{N_{F}}} \frac{(\boldsymbol{p}+\boldsymbol{q}+\boldsymbol{l})_{d} \gamma_{d}}{(\boldsymbol{p}+\boldsymbol{q}+\boldsymbol{l})^{2}} \frac{\gamma_{\epsilon}}{\sqrt{N_{F}}} \frac{(\boldsymbol{p}+\boldsymbol{l})_{e} \gamma_{e}}{(\boldsymbol{p}+\boldsymbol{l})^{2}}\right]\right. \\
+ & \left.N_{F} \operatorname{Tr}\left[\int_{\boldsymbol{l}} \frac{1}{4} \gamma_{\mu}(\boldsymbol{p}+2 \boldsymbol{q}+2 \boldsymbol{l})_{\nu} \frac{(\boldsymbol{q}+\boldsymbol{l})_{c} \gamma_{c}}{(\boldsymbol{q}+\boldsymbol{l})^{2}} \frac{\gamma_{\epsilon}}{\sqrt{N_{F}}} \frac{l_{d} \gamma_{d}}{l^{2}} \frac{\gamma_{\alpha}}{\sqrt{N_{F}}} \frac{(\boldsymbol{p}+\boldsymbol{q}+\boldsymbol{l})_{e} \gamma_{e}}{(\boldsymbol{p}+\boldsymbol{q}+\boldsymbol{l})^{2}}\right]\right)+(\operatorname{perm} 2)
\end{aligned}
$$

Figure 2. Analytical expressions for the diagrams 4 and 6 in figure 5 of the main text. Here, "perm2" indicates permutations $(\mu \leftrightarrow \nu),(\lambda \leftrightarrow \rho)$, and $(\mu \leftrightarrow \nu, \lambda \leftrightarrow \rho)$, and $(\mu \leftrightarrow \lambda, \nu \leftrightarrow \rho)$, switched as a pair. "perm2" will increase the number of terms by a factor of 8 .

\begin{tabular}{|cccc|}
\hline Diagram & $C_{T}^{(i)}$ & Log-Singularity & Factor $a_{i}$ \\
\hline 4 & $-\frac{19}{288 \pi^{2}}$ & $-\frac{1}{24 \pi^{2}} p^{3} \log \frac{\Lambda}{p}$ & 2 \\
6 & $-\frac{1}{128}+\frac{19}{144 \pi^{2}}$ & $\frac{1}{12 \pi^{2}} p^{3} \log \frac{\Lambda}{p}$ & 1 \\
\hline
\end{tabular}

Table 1. Evaluated contributions to the stress tensor correlator and the log-singularities. The log-singularities cancel exactly after summing all graphs.

\section{Acknowledgments}

We thank Simone Giombi, Grigory Tarnopolsky and Igor Klebanov for a correspondence that led to the clarification of the sign error in previous versions of the paper.

Open Access. This article is distributed under the terms of the Creative Commons Attribution License (CC-BY 4.0), which permits any use, distribution and reproduction in any medium, provided the original author(s) and source are credited. 